\title{
Editorial Polling Systems
}

\section{I.J.B.F. Adan • E.M.M. Winands}

Published online: 5 June 2012

(C) The Author(s) 2012. This article is published with open access at Springerlink.com

This special volume of the Annals of Operations Research contains frontier research papers on polling systems. A typical polling system consists of a number of queues, attended by a single server who visits the queues in some order to render service to the customers waiting at the queues. Polling systems naturally arise in a large number of application areas such as maintenance, manufacturing, computer-communication systems and road traffic. In 2000 Hideaki Takagi (2000)—one of the fathers of the success of polling models—wrote the following:

The analysis of polling models gained momentum as queueing systems that are easy to understand, analyze, and extend. The study has been accelerated largely by applications to the modeling of communication, manufacturing and transportation systems. I believe that it is one of the few successful theoretical performance evaluation models developed in the last decades.

In 1992 a special issue was published on polling systems in Queueing Systems, edited by Onno Boxma and Hideaki Takagi. At that time they indicated queueing theory for polling systems to be a presently maturing field. One could wonder whether, almost twenty years since its adolescence phase, the area of polling systems might be going through a mid-life crisis, suffering the well-known symptoms of such a period. Fortunately, nothing is less true! Nowadays, papers on polling systems keep appearing in an unremitting pace.

This special volume identifies the current state-of-the-art in the performance analysis of polling systems and envisages future opportunities and challenges in this research area.

I.J.B.F. Adan ( $₫)$

Department of Mechanical Engineering, Eindhoven University of Technology, P.O. Box 513, 5600 MB

Eindhoven, The Netherlands

e-mail: iadan@tue.nl

E.M.M. Winands

Korteweg-de Vries Institute for Mathematics, University of Amsterdam, Science Park 904, 1098 XH

Amsterdam, The Netherlands

e-mail: e.m.m.winands@uva.nl 
A collection of eight papers is presented, which investigate different research problems in the area of polling systems. This special volume provides an overview of the most recent developments in this area and we hope that it will be an important source of information and inspiration for researchers. A brief preview of the contents of the contributions follows.

Boon studies a polling system with either the exhaustive (a queue must be empty before the server moves on) or gated service discipline (only those customers in the queue at the polling start are served). He introduces the following generalization to this standard polling model: whenever the server starts or ends a visit to a queue, some of the customers waiting in each queue leave the system before having received service. This phenomenon is called reneging (or, alternatively, abandonment or impatience). The cycle time, waiting time, and queue length distributions are analyzed using a generalized version of Little's law.

An extension of the gated service discipline is studied by van der Mei and Roubos, i.e., in this so-called multi-phase gated service discipline each customer that arrives at a queue will have to wait $K_{i}$ cycles before it receives service. Closed-form expressions are derived for the asymptotic waiting time distributions in heavy traffic leading to simple approximations for the moments and tail probabilities of the waiting times under general load. Finally, by using the heavy-traffic results the authors consider the optimization problem of finding the $K_{i}$ that minimizes a weighted sum of the mean waiting times.

In the paper of Al Hanbali et al., the autonomous-server and the time-limited service discipline are studied. An autonomous-server continues servicing a queue for a certain period of time; whereas, in case of a time-limited service discipline, the server continues servicing a queue for a certain period of time or until the queue becomes empty, whichever occurs first. In contrast to the exhaustive and gated service, these two disciplines violate the so-called branching property and the system defies an exact analysis. Therefore, an iterative scheme is introduced that is based on closed-form relations between the joint queue lengths at the beginning and the end of a server visit to a queue.

In addition to the choice of the service discipline (which decides how many customers are served during each visit to a queue) as analyzed in the aforementioned papers, typically a system operator also has to decide in which order customers within each queue are to be served. Hirayama considers an exhaustive or a gated polling model with customer feedback, in which customers are served either first-come-first-served or via a priority queueing discipline. Moreover, in the studied system the server visits the queues according to a Markovian polling order (after service at queue $i$ the server moves to queue $j$ with probability $p_{i, j}$ ). Expressions are obtained for the mean values of various performance measures such as sojourn times, using the functional computation method developed in earlier papers.

Chernova et al. study a polling system with adaptive server routing, i.e., the server routing is dependent on the queue length. For limited service disciplines, the authors show that the stability condition for this system cannot be sharply determined using fluid models that depend only on first moment information. They conjecture that in this case the stability region may depend on the entire distributions of inter-arrival, service, and switch-over times.

The classical assumption of independent switch-over times is relaxed by Fiems and Altman. They investigate a gated polling system in which the switch-over times constitute a sequence of stationary ergodic random variables. In addition, their model includes a Markovian polling order and a random feedback mechanism. By employing the powerful framework of semi-linear equations in a random environment, the authors obtain the first two moments of the workload and queue length at polling instants, and their first moments at random instants.

The above contributions apply to discrete polling systems having a finite number of queues. The papers of Leskelä and Unger and Kavitha and Altman study continuous 
polling systems in which customers arrive according to a Poisson process at random locations on the circle. The former paper considers the setting of a greedy server that polls the circle at random locations sequentially in time, aiming to serve the nearest customer in its neighborhood only and leaving the system unchanged if this neighborhood is empty. The authors show that this system is positive recurrent under the natural stability condition stating that the polling rate should exceed the arrival rate. Their proof is based on a novel quadratic Lyapunov functional that bears the promise of broader applicability. The latter paper does not focus on stability, but aims to analyze the performance of a system where the server is moving continuously on the circle with constant speed, providing globally gated service to some of the customers and exhaustive service to the rest. The central idea of this paper is the use of discretization: the expected virtual workload is obtained as the limit of the expected virtual workload of discretized mixed-service polling systems that obey the wellknown Pseudo conservation laws. These results are then used to obtain relevant performance measures of various configurations of ferry assisted wireless local area networks.

Finally, the above eight papers were chosen for their quality and relevance to the special volume's topic after a highly selective refereeing process. We would like to thank the authors of all submitted papers and the referees for their interest in and support of this special volume.

Open Access This article is distributed under the terms of the Creative Commons Attribution License which permits any use, distribution, and reproduction in any medium, provided the original author(s) and the source are credited.

\section{References}

Takagi, H. (2000). Analysis and application of polling models. In G. Haring, C. Lindemann, \& M. Reiser (Eds.), Lecture notes in computer science: Vol. 1769. Performance evaluation: origins and directions (pp. 424-442). Berlin: Springer. 